\title{
On the values of a class of analytic functions at algebraic points
}

by

\author{
Boris AdAmczewski (Lyon), Yann Bugeaud (Strasbourg) and \\ FLORIAN LUCA (Morelia)
}

1. Introduction. In 1844, Liouville established that the real number $\sum_{k \geq 1} 10^{-k !}$ is transcendental. With a similar method, it is nowadays an easy exercise to extend his result as follows (see [8, p. 2]).

TheOREM L. Let $\alpha$ be an algebraic number with $0<|\alpha|<1$. Then the complex number $\sum_{k \geq 1} \alpha^{k !}$ is transcendental.

In other words, the analytic function $f: z \mapsto \sum_{k \geq 1} z^{k !}$ takes transcendental values at every non-zero algebraic point in its open disc of convergence.

A similar result was obtained by Mahler [6] for a much wider class of functions $f$, including some classical series such as $\mathcal{F}(z)=\sum_{k \geq 0} z^{2^{k}}$ or the Thue-Morse function $\mathcal{T}(z)=\sum_{k \geq 0} t_{k} z^{k}$, where $t_{k}=1$ (resp. $t_{k}=-1$ ) if the sum of digits of the binary expansion of $k$ is even (resp. odd). His method was subsequently refined and generalized by several authors including Kubota, Loxton, van der Poorten, Masser and Nishioka, and the reader is referred to Nishioka's lecture notes [8] for references. Since it always relies on certain functional equations satisfied by the relevant series (for instance, we have $\mathcal{F}\left(z^{2}\right)=\mathcal{F}(z)-z$ and $\left.\mathcal{T}(z)=(1-z) \mathcal{T}\left(z^{2}\right)\right)$, Mahler's method does not allow much flexibility.

The heart of the proof of Theorem L and of Mahler's results is a lower estimate for the distance between two distinct algebraic numbers. Thanks to works by Thue, Siegel, Roth and Schmidt, the seminal result of Liouville has been considerably improved. Thus, it is not surprising that the use of the Schmidt subspace theorem in the present context yields a considerable improvement of Theorem L. This was recently worked out for lacunary functions by Corvaja and Zannier [5], who developed a new approach. We quote

2000 Mathematics Subject Classification: 11J81, 11J61.

Key words and phrases: transcendence, Schmidt subspace theorem. 
below a particular case of Corollary 5 from [5], which is proved by means of a non-trivial application of the Schmidt subspace theorem.

THEOREM CZ. Let $\left(m_{k}\right)_{k \geq 1}$ be an increasing sequence of positive integers such that $\lim \inf _{k} m_{k+1} / m_{k}>1$. Then the function $f: z \mapsto \sum_{k \geq 1} z^{m_{k}}$ takes transcendental values at every algebraic point in the open unit disc.

It is likely that Theorem CZ cannot be proved using Mahler's method or its refinements. Furthermore, the method of [5] offers much more flexibility than Mahler's. Unfortunately, it does not seem to apply easily to non-lacunary functions such as the Thue-Morse function $\mathcal{T}$.

In two earlier works $[3,1]$, we established new transcendence criteria for analytic functions with special combinatorial properties evaluated at the inverse of positive rational integers, Pisot and Salem numbers. These results apply to a broad class of functions including $\mathcal{T}$ and the lacunary series occurring in Theorem CZ (see Section 2 for more details). As in [5], their proofs rest on the Schmidt subspace theorem. It is the aim of the present paper to investigate how our method can be applied to every algebraic point in the open disc of convergence of these analytic functions. Our main result is a general transcendence criterion that extends those of [3] and [1].

2. Stammering functions. In this section, we introduce the notion of stammering function.

Let $\mathcal{A}$ be a countable set. The length of a word $W$ on the alphabet $\mathcal{A}$, that is, the number of letters composing $W$, is denoted by $|W|$. For every positive integer $l$, we write $W^{l}$ for the word $W \ldots W$ (the concatenation of the word $W$ repeated $l$ times). More generally, for every positive real number $x$, we denote by $W^{x}$ the word $W^{\lfloor x\rfloor} W^{\prime}$, where $W^{\prime}$ is the prefix of $W$ of length $\lceil(x-\lfloor x\rfloor)|W|\rceil$. Here, and in all what follows, $\lfloor y\rfloor$ and $\lceil y\rceil$ denote, respectively, the floor and the ceiling of the real number $y$. Let $\mathbf{a}=\left(a_{k}\right)_{k \geq 0}$ be a sequence of elements from $\mathcal{A}$. We say that $\mathbf{a}$ is a stammering sequence if $\mathbf{a}$ is not eventually periodic and if there exist real numbers $w^{\prime} \geq 0$ and $w>1$, and two sequences of finite words $\left(U_{n}\right)_{n \geq 1},\left(V_{n}\right)_{n \geq 1}$ such that:

(i) for any $n \geq 1$, the word $U_{n} V_{n}^{w}$ is a prefix of the word $\mathbf{a}$;

(ii) the sequence $\left(\left|U_{n}\right| /\left|V_{n}\right|\right)_{n \geq 1}$ is bounded from above by $w^{\prime}$;

(iii) the sequence $\left(\left|V_{n}\right|\right)_{n \geq 1}$ is strictly increasing.

Several classical sequences studied in symbolic dynamics, number theory and combinatorics on words turn out to be stammering, as pointed out in [1]. We quote below some famous examples of stammering sequences:

- sequences generated by finite automata (e.g. the Thue-Morse, the Rudin-Shapiro, the Baum-Sweet, and the regular paperfolding sequences); 
- most of the morphic sequences (a morphic sequence is a letter-toletter projection of a fixed point of an endomorphism from a finitely generated free monoid; for instance, the Fibonacci word is the fixed point of the binary morphism $\sigma$ defined by $\sigma(0)=01$ and $\sigma(1)=0$ );

- Sturmian (or Beatty) sequences (i.e., sequences $\left(a_{k}\right)_{k \geq 0}$ such that there exist an irrational real number $\theta$ and a real number $\varrho$ both in $[0,1]$ such that either $a_{k}=\lfloor(k+1) \theta+\varrho\rfloor-\lfloor k \theta+\varrho\rfloor$ for every non-negative integer $k$, or $a_{k}=\lceil(k+1) \theta+\varrho\rceil-\lceil k \theta+\varrho\rceil$ for every non-negative integer $k$ );

- sequences with sublinear block-complexity (these include natural codings of interval exchange transformations, billiard sequences, ArnouxRauzy sequences, ... );

- characteristic sequences of lacunary sets of integers (i.e., sequences $\left(a_{k}\right)_{k \geq 0}$ such that $a_{k}=1$ if $k$ belongs to $\mathbf{m}=\left(m_{l}\right)_{l \geq 1}$ and $a_{k}=0$ otherwise, where $\mathbf{m}$ is an increasing sequence such that $\lim _{\sup } \operatorname{sum}_{l \rightarrow 1} / m_{l}$ $>1$ ).

We refer the reader to [1] and [4] for more details on these sequences.

Throughout the present paper, we denote by $h$ (resp. $H$ ) the logarithmic (resp. multiplicative) absolute height. Their definitions and main properties are given in Section 4. Furthermore, we use the symbols $o, O$ and $\ll$ with their usual meanings.

We say that a sequence of algebraic numbers $\left(a_{k}\right)_{k \geq 0}$ satisfies the growth condition $(G)$ if either

$$
h\left(a_{0}, \ldots, a_{n}\right)=o(n) \quad \text { as } n \rightarrow \infty,
$$

or

$$
h\left(a_{k}\right) \ll 1 .
$$

Obviously, by (2.2), every bounded sequence of rational integers satisfies the growth condition $(G)$.

With a stammering sequence $\mathbf{a}=\left(a_{k}\right)_{k \geq 0}$ of algebraic numbers in a number field $\mathbb{L}$ that satisfies the growth condition $(G)$, we associate the analytic function $f_{\mathbf{a}}$ defined on the open unit disc by

$$
f_{\mathbf{a}}(z)=\sum_{k=0}^{+\infty} a_{k} z^{k}
$$

Such a function is called a stammering function. This notation will be kept throughout the entire paper. We note that all the series mentioned in Section 1 are stammering ones.

3. Results. We are interested in values of stammering functions at algebraic points. In view of the results mentioned previously, it is likely that 
such functions essentially take transcendental values at algebraic points. More precisely, we can expect the following picture: given a stammering function $f_{\mathbf{a}}$ as in (2.3), then $f_{\mathbf{a}}(\alpha)$ is transcendental for all but finitely many algebraic numbers $\alpha$ lying in the open disc of convergence of $f_{\mathbf{a}}$, and the algebraic values of $f_{\mathbf{a}}$ belong to the number field $\mathbb{L}(\alpha)$.

A first result towards this problem was proved in [3].

Theorem ABL. Let $b \geq 2$ be a rational integer. If $\mathbf{a}=\left(a_{k}\right)_{k \geq 0}$ is a stammering sequence on the set $\{0,1, \ldots, b-1\}$, then the stammering function $f_{\mathbf{a}}$ takes transcendental values at the inverse of every positive rational integer.

As worked out in [1], this transcendence criterion is powerful enough to confirm the Cobham-Loxton-van der Poorten conjecture claiming that the $b$-adic expansion of an algebraic irrational real number cannot be generated by a finite automaton. The above is a result that seems to fall outside Mahler's method.

Subsequently, Theorem ABL was extended in [1] in the following way. Recall that a Pisot (resp. Salem) number is a real algebraic integer $>1$ whose complex conjugates lie inside the open unit disc (resp. inside the closed unit disc, with at least one of them on the unit circle).

Theorem AB. Let $\mathbf{a}=\left(a_{k}\right)_{k \geq 0}$ be a stammering, bounded sequence of rational integers. If $\beta$ is a Pisot or a Salem number, then $f_{\mathbf{a}}(1 / \beta)$ is either transcendental, or belongs to the number field $\mathbb{Q}(\beta)$.

Note that we cannot avoid the possibility that $f_{\mathbf{a}}(1 / \beta)$ belongs to $\mathbb{Q}(\beta)$. Indeed, let $\beta=(\sqrt{5}+1) / 2$ be the golden ratio. Then, starting with a periodic sequence $\mathbf{a}=\left(a_{k}\right)_{k \geq 1}$, we can use the fact that $1=1 / \beta+1 / \beta^{2}$ to construct (using only local perturbation of the sequence $\mathbf{a}$ ) a stammering sequence $\mathbf{a}^{\prime}=\left(a_{k}^{\prime}\right)_{k \geq 0}$ such that

$$
\mathbb{Q}(\beta) \ni \sum_{k=0}^{+\infty} \frac{a_{k}}{\beta^{k}}=\sum_{k=0}^{+\infty} \frac{a_{k}^{\prime}}{\beta^{k}} .
$$

The proof of Theorem AB rests on a $p$-adic version due to Schlickewei [9] of the Schmidt subspace theorem. The crucial point to apply the subspace theorem is that the linear form $f_{\mathbf{a}}(\alpha) X-f_{\mathbf{a}}(\alpha) Y-Z$ takes small values at many algebraic points when $\alpha:=1 / \beta$ is the inverse of a Pisot or a Salem number. Unfortunately, when $\alpha$ is an arbitrary complex algebraic number lying in the open unit disc, the values taken by this linear form are no longer small enough with respect to the height of the relevant algebraic points. Notice also that a similar difficulty explains the condition imposed on the constant $L$ in Theorem 3 of Corvaja and Zannier [5]. 
If $|\cdot|_{j}$ is an Archimedean absolute value given by the embedding $\sigma_{j}$ such that $|\alpha|_{j}<1$, then we can consider the complex number $f_{\mathbf{a}, j}(\alpha)$ defined by

$$
f_{\mathbf{a}, j}(\alpha):=\lim _{N \rightarrow+\infty} \sum_{k=0}^{N} a_{k} \sigma_{j}\left(\alpha^{k}\right) .
$$

Again, if $\mathfrak{p}$ is a finite place such that $|\alpha|_{\mathfrak{p}}<1$, then we can consider in the $\mathfrak{p}$-adic completion of the field $\mathbb{Q}(\beta)$ the number $f_{\mathbf{a}, \mathfrak{p}}(\alpha)$ defined by

$$
f_{\mathbf{a}, \mathfrak{p}}(\alpha):=\lim _{N \rightarrow+\infty} \sum_{k=0}^{N} a_{k} \alpha^{k} .
$$

The main novelty of the present paper is to remark that all the linear forms $f_{\mathbf{a}, j}(\alpha) X-f_{\mathbf{a}, j}(\alpha) Y-Z$ and $f_{\mathbf{a}, \mathfrak{p}}(\alpha) X-f_{\mathbf{a}, \mathfrak{p}}(\alpha) Y-Z$ also take small values at many algebraic points constructed in the same way as in the proof of Theorem AB. Actually, when evaluated at such points the product of all linear forms we consider is just small enough to apply the subspace theorem.

Our main result is the following extension of Theorem AB to any nonzero complex algebraic number lying in the open unit disc.

TheOREM 1. Let $\mathbf{a}=\left(a_{k}\right)_{k \geq 0}$ be a stammering sequence of algebraic numbers in a number field $\mathbb{L}$ and suppose that a satisfies (2.1) or (2.2). Let $\alpha$ be a non-zero algebraic number lying in the open unit disc and assume that $\alpha_{1}=\alpha, \alpha_{2}, \ldots, \alpha_{l}$ are all its complex conjugates of modulus $<1$. Let $\mathfrak{p}_{1}, \ldots, \mathfrak{p}_{l^{\prime}}$ be all the prime ideals in $\mathbb{L}(\alpha)$ such that $|\alpha|_{\mathfrak{p}_{j}}<1$. Then either (at least) one of the numbers $f_{\mathbf{a}, 1}(\alpha):=f_{\mathbf{a}}(\alpha), f_{\mathbf{a}, 2}(\alpha), \ldots, f_{\mathbf{a}, l}(\alpha)$, $f_{\mathbf{a}, \mathfrak{p}_{1}}(\alpha), \ldots, f_{\mathbf{a}, \mathfrak{p}_{l^{\prime}}}(\alpha)$ is transcendental, or $f_{\mathbf{a}, j}(\alpha)$ belongs to $\mathbb{L}\left(\alpha_{j}\right)$ for $j=$ $1, \ldots, l$. Furthermore, in this last case, if we let $\sigma_{j}$ be some automorphism of the Galois closure of $\mathbb{L}(\alpha)$ over $\mathbb{Q}$ sending $\alpha$ to $\alpha_{j}$, then $f_{\mathbf{a}, j}(\alpha)=\sigma_{j}\left(f_{\mathbf{a}}(\alpha)\right)$ for all $j=2, \ldots, l$.

We stress that Theorem 1 plainly includes Theorems ABL and AB. The proof of Theorem 1 also rests on the $p$-adic version of the Schmidt subspace theorem given in [9]. The main interest of Theorem 1 is that it applies to every algebraic value of every stammering function. However, the price to pay for this (i.e., the introduction of new linear forms) produces a weaker conclusion than the one expected. We mention that another way to proceed, worked out in [2], yields a conclusion as strong as in Theorem AB, but the price to pay is then a strengthening of the stammering condition on $\mathbf{a}$.

We now give two straightforward consequences of Theorem 1 .

Corollary 1. Let $\mathbf{a}=\left(a_{k}\right)_{k \geq 0}$ be a stammering sequence of algebraic numbers in a number field $\mathbb{L}$ and suppose that a satisfies (2.1) or (2.2). Let $\beta$ be an algebraic integer such that $|\beta|>1$ and assume that $\beta_{1}=\beta, \beta_{2}, \ldots, \beta_{l}$ 
are all its complex conjugates of modulus $>1$. If all the complex numbers

$$
\gamma_{j}:=\sum_{k=0}^{+\infty} \frac{a_{k}}{\beta_{j}^{k}}, \quad 1 \leq j \leq l,
$$

are algebraic, then $\gamma_{j}$ belongs to the number field $\mathbb{L}\left(\beta_{j}\right)$ for $j=1, \ldots, l$.

Corollary 2. Let $\mathbf{a}=\left(a_{k}\right)_{k \geq 0}$ be a bounded, stammering sequence of rational integers. Let $p$ and $q$ be positive coprime integers with $q>p$ and $p$ prime. If both the real number

$$
\gamma:=\sum_{k=0}^{+\infty} a_{k}\left(\frac{p}{q}\right)^{k}
$$

and the p-adic number

$$
\sum_{k=0}^{+\infty} a_{k}\left(\frac{p}{q}\right)^{k}
$$

are algebraic, then $\gamma$ is rational.

We end this section with an application of Theorem 1 to the expansion of algebraic numbers in a complex quadratic integer base $b$, which in turn has an amusing application to the transcendence of alternating stammering numbers.

THEOREM 2. Let $b$ be an algebraic integer in an imaginary quadratic field with $|b|>1$. Let $\mathbf{a}=\left(a_{k}\right)_{k \geq 0}$ be a stammering sequence with values in the set $\left\{0,1, \ldots,|b|^{2}-1\right\}$. Then the number

$$
\sum_{k=0}^{+\infty} a_{k} / b^{k}
$$

is transcendental.

Corollary 3. If $b>1$ is a positive integer and $\mathbf{a}=\left(a_{k}\right)_{k \geq 0}$ is a stammering sequence taking its values in the set $\{0,1, \ldots, b-1\}$, then the number

$$
\sum_{k=0}^{+\infty}(-1)^{k} a_{k} / b^{k}
$$

is transcendental.

4. The auxiliary result. Our main auxiliary tool is a version of the $\mathfrak{P}$-adic Thue-Siegel-Roth-Schmidt theorem proved by Schlickewei [9]. We make use of the following notation (see also [7, pp. 182-183]).

Let $p$ be a prime number. Let $\mathbb{Q}_{p}$ be the completion of $\mathbb{Q}$ at $p$ and $\mathbb{C}_{p}$ the completion of the algebraic closure of $\mathbb{Q}_{p}$ for the absolute value $|\cdot|_{p}$. 
Let $\mathbb{K}$ be a number field of degree $d$. Write the ideal $(p)$ in $\mathcal{O}_{\mathbb{K}}$ as

$$
(p)=\prod_{j=1}^{\pi(p)} \mathfrak{p}_{j}^{e\left(\mathfrak{p}_{j}\right)}
$$

where $\mathfrak{p}_{1}, \ldots, \mathfrak{p}_{\pi(p)}$ are distinct prime ideals in $\mathcal{O}_{\mathbb{K}}$. For a non-zero element $\alpha$ in $\mathbb{K}$, set

$$
|\alpha|_{\mathfrak{p}_{j}}=p^{-h_{j} / e\left(\mathfrak{p}_{j}\right)},
$$

where $h_{j}$ is the largest integer such that $\mathfrak{p}_{j}$ is coprime with the fractional ideal $\mathfrak{p}_{j}^{h_{j}} \times(\alpha)$. For $j=1, \ldots, \pi(p)$, let $d\left(\mathfrak{p}_{j}\right)$ denote the degree of the completion $\mathbb{K}_{\mathfrak{p}_{j}}$ of $\mathbb{K}$ with respect to $\mathfrak{p}_{j}$. Let $r$ be the unit rank of $\mathbb{K}$. For $j=1, \ldots, r+1$, let $d_{j}$ equal to 1 (resp. 2 ) if the embedding of $\mathbb{K}$ associated with the absolute value $\mid \cdot{ }_{j}$ is real (resp. complex).

For any non-zero $\alpha$ in $\mathbb{K}$, we have the product formula

$$
\prod_{j=1}^{r+1}|\alpha|_{j}^{d_{j}} \cdot \prod_{\mathfrak{p}}|\alpha|_{\mathfrak{p}}^{d(\mathfrak{p})}=1 .
$$

The field height $H_{\mathbb{K}}$ of $\underline{\beta}=\left(\beta_{0}, \ldots, \beta_{m}\right) \in \mathbb{K}^{m+1}$ is defined by

$$
H_{\mathbb{K}}(\underline{\beta})=\prod_{j=1}^{r+1} \max \left\{\left|\beta_{0}\right|_{j}^{d_{j}}, \ldots,\left|\beta_{m}\right|_{j}^{d_{j}}\right\} \cdot \prod_{\mathfrak{p}} \max \left\{\left|\beta_{0}\right|_{\mathfrak{p}}^{d(\mathfrak{p})}, \ldots,\left|\beta_{m}\right|_{\mathfrak{p}}^{d(\mathfrak{p})}\right\},
$$

where the second product is taken over all inequivalent non-Archimedean primes of $\mathbb{K}$.

Let $p_{0}=\infty$ denote the Archimedean prime in $\mathbb{Q}$. Denote by $\mathbb{K}_{1}^{(0)}, \ldots, \mathbb{K}_{r+1}^{(0)}$ the $r+1$ non-isomorphic fields corresponding to the embeddings of $\mathbb{K}$ into $\mathbb{C}$. For simplicity, set $\pi\left(p_{0}\right)=r+1$. Let $\left\{p_{1}, \ldots, p_{t}\right\}$ be a finite set of rational prime numbers. For $i=1, \ldots, t$, denote by $\mathbb{K}_{1}^{(i)}, \ldots, \mathbb{K}_{\pi\left(p_{i}\right)}^{(i)}$ the $\pi\left(p_{i}\right)$ pairwise non-isomorphic fields corresponding to the embeddings of $\mathbb{K}$ into $\mathbb{C}_{p_{i}}$. Furthermore, we denote the images of an $(m+1)$-tuple $\underline{\beta}=\left(\beta_{0}, \ldots, \beta_{m}\right) \in \mathbb{K}^{m+1}$ under these embeddings as follows: for $i=0, \ldots, \bar{t}$ and for $j=1, \ldots, \pi\left(p_{i}\right)$, we write $\underline{\beta}_{j}^{(i)}=\left(\beta_{0 j}^{(i)}, \ldots, \beta_{m j}^{(i)}\right) \in \mathbb{K}_{j}^{(i)}{ }^{m+1}$.

Furthermore, for $j=1, \ldots, r+1$, set

$$
\left|\underline{\beta}_{j}^{(0)}\right|=\max \left\{\left|\beta_{0 j}^{(0)}\right|, \ldots,\left|\beta_{m j}^{(0)}\right|\right\},
$$

and, for $i=1, \ldots, t$ and $j=1, \ldots, \pi\left(p_{i}\right)$, set

$$
\left|\underline{\beta}_{j}^{(i)}\right|_{\mathfrak{p}_{i j}}=\max \left\{\left|\beta_{0 j}^{(i)}\right|_{\mathfrak{p}_{i j}}, \ldots,\left|\beta_{m j}^{(i)}\right|_{\mathfrak{p}_{i j}}\right\} .
$$

With these notations, Theorem 2.1 of [9] combined with Lemma 1F, page 178 of [10] yields the following version of the subspace theorem, which is the key tool for the proof of our Theorem 1. 
Theorem S. For every $i=0, \ldots, t$ and every $j=1, \ldots, \pi\left(p_{i}\right)$, let $L_{0 j}^{(i)}, \ldots, L_{m j}^{(i)}$ be $m+1$ linearly independent linear forms in $m+1$ variables with algebraic coefficients from the field $\mathbb{C}_{p_{i}}$. Then, for any $\varepsilon>0$, all the solutions $\underline{\beta} \in \mathbb{P}^{m}(\mathbb{K})$ to the inequality

$$
\prod_{j=1}^{r+1} \prod_{k=0}^{m} \frac{\left|L_{k j}^{(0)}\left(\underline{\beta}_{j}^{(0)}\right)\right|^{d_{j}}}{\left|\underline{\beta}_{j}^{(0)}\right|^{d_{j}}} \cdot \prod_{i=1}^{t} \prod_{j=1}^{\pi\left(p_{i}\right)} \prod_{k=0}^{m} \frac{\left|L_{k j}^{(i)}\left(\underline{\beta}_{j}^{(i)}\right)\right|_{\mathfrak{p}_{i j}}^{d\left(\mathfrak{p}_{i j}\right)}}{\left|\underline{\beta}_{j}^{(i)}\right|_{\mathfrak{p}_{i j}}^{d\left(\mathfrak{p}_{i j}\right)}} \leq H_{\mathbb{K}}(\underline{\beta})^{-m-1-\varepsilon}
$$

lie in finitely many proper subspaces.

We end this section with a few words on heights. Keep the above notation and let $\beta$ be in $\mathbb{K}$. The (multiplicative) absolute height of $\beta$ is defined by

$$
H(\beta):=\left(H_{\mathbb{K}}((1, \beta))\right)^{1 /[\mathbb{K}: \mathbb{Q}]},
$$

and we set

$$
h(\beta)=\log H(\beta) .
$$

We refer to Chapter 3 of [11] for classical results on these heights.

We just mention that, for any algebraic numbers $a_{0}, \ldots, a_{n}, \beta$ in $\mathbb{K}$ and for any place $\nu$ in $\mathbb{K}$, we have

$$
\begin{aligned}
\left|a_{0}+a_{1} \beta+\cdots+a_{n} \beta^{n}\right|_{\nu} & \\
& \leq \max \left\{1,|n+1|_{\nu}\right\} \cdot \max \left\{\left|a_{0}\right|_{\nu},\left|a_{1} \beta\right|_{\nu}, \ldots,\left|a_{n} \beta^{n}\right|_{\nu}\right\} \\
& \leq \max \left\{1,|n+1|_{\nu}\right\} \cdot \max \left\{\left|a_{0}\right|_{\nu}, \ldots,\left|a_{n}\right|_{\nu}\right\} \cdot \max \left\{1,|\beta|_{\nu}\right\}^{n}
\end{aligned}
$$

and

$$
h\left(a_{0}, \ldots, a_{n}\right):=\frac{\log \left(H_{\mathbb{K}}\left(\left(a_{0}, \ldots, a_{n}\right)\right)\right)}{[\mathbb{K}: \mathbb{Q}]}
$$

5. Proof of Theorem 1. Consider a stammering sequence $\mathbf{a}=\left(a_{k}\right)_{k \geq 0}$ of algebraic numbers in a number field $\mathbb{L}$ that satisfies $(2.1)$ or $(2.2)$. We assume that the parameters $w$ and $w^{\prime}$ are fixed, as are the sequences $\left(U_{n}\right)_{n \geq 1}$ and $\left(V_{n}\right)_{n \geq 1}$ occurring in the definition of a stammering sequence. Set also $r_{n}=\left|U_{n}\right|$ and $s_{n}=\left|V_{n}\right|$ for every $n \geq 1$. Observe that $s_{n}$ tends to infinity monotonically with $n$.

We fix a complex algebraic number $\alpha$ in the open unit disc and set $\beta:=1 / \alpha$. We point out that $|\beta|>1$. Set $\mathbb{K}:=\mathbb{L}(\alpha)=\mathbb{L}(\beta)$ and $\delta=$ $[\mathbb{K}: \mathbb{Q}] /[\mathbb{Q}(\beta): \mathbb{Q}]$. Assume that the conjugates of $\beta$ are numbered in such a way that $\beta=\beta_{1}, \ldots, \beta_{l}$ is a subset of the conjugates of $\beta$ of modulus $>1$ containing exactly one of the two elements of each pair of complex conjugates.

For any $j=1, \ldots, l$, set

$$
f_{\mathbf{a}, j}(\alpha):=f_{\mathbf{a}}\left(\alpha_{j}\right)=\sum_{k=0}^{+\infty} a_{k} \alpha_{j}^{k}=\sum_{k=0}^{+\infty} \frac{a_{k}}{\beta_{j}^{k}} .
$$


Let $\mathfrak{p}_{1}, \ldots, \mathfrak{p}_{l^{\prime}}$ be the prime ideals $\mathfrak{p}$ in $\mathbb{K}$ with $|\alpha|_{\mathfrak{p}}<1$ (i.e., with $|\beta|_{\mathfrak{p}}>1$ ). For $j=1, \ldots, l^{\prime}$, let $f_{\mathbf{a}, \mathfrak{p}_{j}}(\alpha)$ in $\mathbb{K}_{\mathfrak{p}_{j}}$ be defined by

$$
f_{\mathbf{a}, \mathfrak{p}_{j}}(\alpha):=\lim _{N \rightarrow+\infty} \sum_{k=0}^{N} \frac{a_{k}}{\beta^{k}} .
$$

The key fact is the observation that the $f_{\mathbf{a}, j}(\alpha)$ 's and the $f_{\mathbf{a}, \mathfrak{p}_{j}}(\alpha)$ 's defined above admit infinitely many good approximants in the fields $\mathbb{K}$ and $\mathbb{K}_{\mathfrak{p}_{j}}$, respectively. These approximants are obtained by truncating their associated series and completing them by periodicity. Precisely, for every positive integer $n$, we define the sequence $\left(b_{k}^{(n)}\right)_{k \geq 0}$ by

$$
\begin{aligned}
\quad b_{h}^{(n)} & =a_{h} & & \text { for } 0 \leq h \leq r_{n}+s_{n}-1, \\
b_{r_{n}+h+j s_{n}}^{(n)} & =a_{r_{n}+h} & & \text { for } 0 \leq h \leq s_{n}-1 \text { and } j \geq 0 .
\end{aligned}
$$

The sequence $\left(b_{k}^{(n)}\right)_{k \geq 0}$ is eventually periodic, with preperiod $U_{n}$ and with period $V_{n}$. For $j=1, \ldots, l$, set

$$
\alpha_{j}^{(n)}=\sum_{k=0}^{+\infty} \frac{b_{k}^{(n)}}{\beta_{j}^{k}} .
$$

Since $\left(a_{k}\right)_{k \geq 0}$ satisfies (2.1) or (2.2), we have $\left|a_{k}-b_{k}^{(n)}\right| \ll c^{k}$ for every real number $c>1$. Throughout the proof of the theorem, all the constants implied by $\ll$ may depend on $c$, but they are independent of $n$. We thus observe that

$$
\left|f_{\mathbf{a}, j}(\alpha)-\alpha_{j}^{(n)}\right|=\left|\sum_{k=r_{n}+\left\lceil w s_{n}\right\rceil}^{+\infty} \frac{a_{k}-b_{k}^{(n)}}{\beta_{j}^{k}}\right| \ll \frac{c^{r_{n}+\left\lceil w s_{n}\right\rceil}}{\left|\beta_{j}\right|^{r_{n}+\left\lceil w s_{n}\right\rceil}}
$$

for every real number $c>1$. Likewise, for $j=1, \ldots, l^{\prime}$, define $\alpha_{\mathfrak{p}_{j}}^{(n)}$ in $\mathbb{K}_{\mathfrak{p}_{j}}$ by the $p$-adic limit

$$
\alpha_{\mathfrak{p}_{j}}^{(n)}=\lim _{N \rightarrow+\infty} \sum_{k=0}^{N} \frac{b_{k}^{(n)}}{\beta^{k}}
$$

and observe that

$$
\left|f_{\mathbf{a}, \mathfrak{p}_{j}}(\alpha)-\alpha_{\mathfrak{p}_{j}}^{(n)}\right|_{\mathfrak{p}_{j}} \ll \frac{c^{r_{n}+\left\lceil w s_{n}\right\rceil}}{|\beta|_{\mathfrak{p}_{j}}^{r_{n}+\left\lceil w s_{n}\right\rceil}}
$$

for every real number $c>1$.

Lemma 1. For every integer $n$, set

$$
P_{n}(X)=\sum_{k=0}^{r_{n}-1} a_{k} X^{r_{n}-k}\left(X^{s_{n}}-1\right)+\sum_{k=0}^{s_{n}-1} a_{r_{n}+k} X^{s_{n}-k} .
$$


Then, for $j=1, \ldots, l$, we have

$$
\alpha_{j}^{(n)}=\frac{P_{n}\left(\beta_{j}\right)}{\beta_{j}^{r_{n}}\left(\beta_{j}^{s_{n}}-1\right)} .
$$

Proof. This is an easy verification, as in Lemma 1 from [1].

Now, we define the systems of linear forms to which we will apply Theorem S. Let us assume that $f_{\mathbf{a}, 1}(\alpha):=f_{\mathbf{a}}(\alpha), \ldots, f_{\mathbf{a}, l}(\alpha)$ and $f_{\mathbf{a}, \mathfrak{p}_{1}}(\alpha), \ldots$ $\ldots, f_{\mathbf{a}, \mathfrak{p}_{l^{\prime}}}(\alpha)$ are all algebraic.

For $j=1, \ldots, l$, consider the linear forms

$$
L_{0 j}^{(0)}=X, \quad L_{1 j}^{(0)}=Y, \quad L_{2 j}^{(0)}=f_{\mathbf{a}, j}(\alpha) X-f_{\mathbf{a}, j}(\alpha) Y-Z .
$$

For the remaining Archimedean absolute values, that is, for $j=l+1, \ldots, r+1$, where $r$ denotes the unit rank of $\mathbb{Q}(\beta)$, take simply

$$
L_{0 j}^{(0)}=X, \quad L_{1 j}^{(0)}=Y, \quad L_{2 j}^{(0)}=Z .
$$

Let $p_{1}, \ldots, p_{t}$ be the prime numbers $p$ such that there exists an ideal $\mathfrak{p}$ above $p$ with $|\beta|_{\mathfrak{p}} \neq 1$. Each of the prime ideals $\mathfrak{p}_{1}, \ldots, \mathfrak{p}_{l^{\prime}}$ divides one of the $p_{i}$ 's. For $j=1, \ldots, l^{\prime}$, let $p_{i}$ be the rational prime above $\mathfrak{p}_{j}$ and consider the linear forms

$$
L_{0 j}^{(i)}=X, \quad L_{1 j}^{(i)}=Y, \quad L_{2 j}^{(i)}=f_{\mathbf{a}, \mathfrak{p}_{j}}(\alpha) X-f_{\mathbf{a}, \mathfrak{p}_{j}}(\alpha) Y-Z .
$$

For all the other prime ideals $\mathfrak{p}$ below one of the $p_{i}$ 's, we consider the linear forms

$$
L_{0 j}^{(i)}=X, \quad L_{1 j}^{(i)}=Y, \quad L_{2 j}^{(i)}=Z .
$$

We evaluate the product of these linear forms at the algebraic points $\underline{\beta}_{j}^{(i)}$, where

$$
\underline{\beta}=\left(\beta^{r_{n}+s_{n}}, \beta^{r_{n}}, P_{n}(\beta)\right) .
$$

Obviously, $\beta$ and the $\underline{\beta}_{j}^{(i)}$ 's depend on $n$; however, we choose not to indicate this dependence for sake of simplicity.

We first establish an upper bound for the height of $\underline{\beta}$.

Lemma 2. For every real number $c>1$, we have

$$
H_{\mathbb{K}}(\underline{\beta}) \ll\left(c \prod_{j=1}^{l}\left|\beta_{j}\right|^{d_{j}} \cdot \prod_{j=1}^{l^{\prime}}|\beta|_{\mathfrak{p}_{j}}^{d_{\mathfrak{p}_{j}}}\right)^{r_{n}+s_{n}},
$$

where the constant implied by the Vinogradov symbol $\ll$ is independent of $n$. 
Proof. Let $c>1$ be arbitrary. By definition,

$$
\begin{aligned}
H_{\mathbb{K}}(\underline{\beta})= & \prod_{j=1}^{r+1} \max \left\{\left|\beta_{j}^{r_{n}+s_{n}}\right|^{d_{j}},\left|\beta_{j}^{r_{n}}\right|^{d_{j}},\left|P_{n}\left(\beta_{j}\right)\right|^{d_{j}}\right\} \\
& \cdot \prod_{\mathfrak{p}} \max \left\{\left|\beta^{r_{n}+s_{n}}\right|_{\mathfrak{p}}^{d(\mathfrak{p})},\left|\beta^{r_{n}}\right|_{\mathfrak{p}}^{d(\mathfrak{p})},\left|P_{n}(\beta)\right|_{\mathfrak{p}}^{d(\mathfrak{p})}\right\} .
\end{aligned}
$$

On the one hand, we infer from the fact that a satisfies (2.1) or (2.2) and from Lemma 1 that

$$
\begin{aligned}
\prod_{j=1}^{r+1} \max \left\{\left.\left|\beta_{j}^{r_{n}+s_{n}}\right|\right|^{d_{j}},\left|\beta_{j}^{r_{n}}\right|^{d_{j}},\left|P_{n}\left(\beta_{j}\right)\right|^{d_{j}}\right\} & \\
& \ll\left(\prod_{j=1}^{l}\left|\beta_{j}\right|^{d_{j}}\right)^{r_{n}+s_{n}} \cdot \prod_{j=l+1}^{r+1} \max \left\{1,\left|P_{n}\left(\beta_{j}\right)\right|^{d_{j}}\right\} \\
& \ll\left(\prod_{j=1}^{l}\left|\beta_{j}\right|^{d_{j}}\right)^{r_{n}+s_{n}} c^{\left(r_{n}+s_{n}\right) / 2} .
\end{aligned}
$$

On the other hand, we have

$$
\begin{aligned}
\prod_{\mathfrak{p}} \max \left\{\left|\beta^{r_{n}+s_{n}}\right|_{\mathfrak{p}}^{d(\mathfrak{p})},\left|\beta^{r_{n}}\right|_{\mathfrak{p}}^{d(\mathfrak{p})}, \mid\right. & \left.\left.P_{n}(\beta)\right|_{\mathfrak{p}} ^{d(\mathfrak{p})}\right\} \\
& \ll\left(\prod_{j=1}^{l^{\prime}}|\beta|_{\mathfrak{p}_{j}}^{d\left(\mathfrak{p}_{j}\right)}\right)^{r_{n}+s_{n}} \cdot \prod_{\mathfrak{p} \in \mathcal{P}}\left|P_{n}(\beta)\right|_{\mathfrak{p}}^{d(\mathfrak{p})},
\end{aligned}
$$

where the latter product is taken over the set $\mathcal{P}$ of all the prime ideals $\mathfrak{p}$ that do not divide $\mathfrak{p}_{1} \ldots \mathfrak{p}_{l^{\prime}}$ and are such that $\left|P_{n}(\beta)\right|_{\mathfrak{p}}>1$. Furthermore, we have

$$
\prod_{\mathfrak{p} \in \mathcal{P}}\left|P_{n}(\beta)\right|_{\mathfrak{p}}^{d(\mathfrak{p})} \ll c^{\left(r_{n}+s_{n}\right) / 2} .
$$

Indeed, we infer from (2.1), inequality (4.2), Lemma 1 and inequality (4.3) that

$$
\begin{aligned}
\prod_{\mathfrak{p} \in \mathcal{P}} \mid P_{n} & \left.(\beta)\right|_{\mathfrak{p}} ^{d(\mathfrak{p})} \\
& \leq \prod_{\mathfrak{p} \in \mathcal{P}}\left(\max \left\{1,\left|r_{n}+s_{n}\right|_{\mathfrak{p}}^{d(\mathfrak{p})}\right\} \cdot \max \left\{\left|a_{0}\right|_{\mathfrak{p}}^{d(\mathfrak{p})}, \ldots,\left|a_{r_{n}+s_{n}-1}\right|_{\mathfrak{p}}^{d(\mathfrak{p})}\right\}\right) \\
& \leq\left(r_{n}+s_{n}\right) H_{\mathbb{K}}\left(a_{0}, \ldots, a_{r_{n}+s_{n}-1}\right) \\
& \ll\left(r_{n}+s_{n}\right) \exp \left\{[\mathbb{K}: \mathbb{Q}] \cdot h\left(a_{0}, \ldots, a_{r_{n}+s_{n}-1}\right)\right\} \ll c^{\left(r_{n}+s_{n}\right) / 2} .
\end{aligned}
$$

The same upper bound holds under the assumption (2.2) since, in this case, 
the product

$$
\prod_{\mathfrak{p} \in \mathcal{P}} \max \left\{\left|a_{0}\right|_{\mathfrak{p}}^{d(\mathfrak{p})}, \ldots,\left|a_{r_{n}+s_{n}-1}\right|_{\mathfrak{p}}^{d(\mathfrak{p})}\right\}
$$

is bounded by a constant independent of $n$. This ends the proof.

Observe that our choice of $p_{1}, \ldots, p_{t}$ implies that $|\underline{\beta}|_{\mathfrak{p}}=1$ for any prime $\mathfrak{p}$ which does not lie above one of the $p_{i}$ 's. Consequently, we get

$$
\prod_{j=1}^{r+1}\left|\underline{\beta}_{j}^{(0)}\right|^{d_{j}} \cdot \prod_{i=1}^{t} \prod_{j=1}^{\pi\left(p_{i}\right)}\left|\underline{\beta}_{j}^{(i)}\right|_{\mathfrak{p}_{i j}}^{d\left(\mathfrak{p}_{i j}\right)}=H_{\mathbb{K}}(\underline{\beta}) \cdot \prod_{\mathfrak{p} \in \mathcal{P}^{\prime}}\left|P_{n}(\beta)\right|_{\mathfrak{p}}^{-d(\mathfrak{p})},
$$

where the latter product is taken over the set $\mathcal{P}^{\prime}$ of all the prime ideals $\mathfrak{p}$ that do not divide $p_{1} \ldots p_{t}$ and are such that $\left|P_{n}(\beta)\right|_{\mathfrak{p}}>1$. Obviously, we infer from (5.3) that

$$
\prod_{\mathfrak{p} \in \mathcal{P}^{\prime}}\left|P_{n}(\beta)\right|_{\mathfrak{p}}^{d(\mathfrak{p})} \leq \prod_{\mathfrak{p} \in \mathcal{P}}\left|P_{n}(\beta)\right|_{\mathfrak{p}}^{d(\mathfrak{p})} \ll c^{\left(r_{n}+s_{n}\right) / 2}
$$

for every real number $c>1$.

By the product formula, we have

$$
\prod_{j=1}^{r+1}\left|L_{k j}^{(0)}\left(\underline{\beta}_{j}^{(0)}\right)\right|^{\delta d_{j}} \cdot \prod_{i=1}^{t} \prod_{j=1}^{\pi\left(p_{i}\right)}\left|L_{k j}^{(i)}\left(\underline{\beta}_{j}^{(i)}\right)\right|_{\mathfrak{p}_{i j}}^{d\left(\mathfrak{p}_{i j}\right)}=1
$$

for $k=0,1$.

It remains to evaluate the expressions $\left|L_{2 j}^{(i)}\left(\underline{\beta}_{j}^{(i)}\right)\right|$. By (5.1), the Archimedean places yield a contribution of at most

$$
\prod_{j=1}^{r+1}\left|L_{2 j}^{(0)}\left(\underline{\beta}_{j}^{(0)}\right)\right|^{\delta d_{j}} \ll c_{0}^{r_{n}+s_{n}} \prod_{j=1}^{l}\left|\beta_{j}\right|^{-\delta d_{j}(w-1) s_{n}}
$$

for a certain real number $c_{0}>1$ that will be selected later on to be sufficiently close to 1 . By (5.2), the contribution of the non-Archimedean places yields

$$
\prod_{i=1}^{t} \prod_{j=1}^{\pi\left(p_{i}\right)}\left|L_{2 j}^{(i)}\left(\underline{\beta}_{j}^{(i)}\right)\right|_{\mathfrak{p}_{i j}}^{d\left(\mathfrak{p}_{i j}\right)} \ll c_{0}^{r_{n}+s_{n}} \prod_{j=1}^{l^{\prime}}|\beta|_{\mathfrak{p}_{j}}^{-(w-1) s_{n} d\left(\mathfrak{p}_{j}\right)} .
$$

Consequently, choosing $c=c_{0}^{1 / 3}$ in (5.5), we infer from (5.4) and (5.6)(5.8) that the product

$$
\Pi:=\prod_{j=1}^{r+1} \prod_{k=0}^{2} \frac{\left|L_{k j}^{(0)}\left(\underline{\beta}_{j}^{(0)}\right)\right|^{d_{j}}}{\left|\underline{\beta}_{j}^{(0)}\right|^{d_{j}}} \cdot \prod_{i=1}^{t} \prod_{j=1}^{\pi\left(p_{i}\right)} \prod_{k=0}^{2} \frac{\left|L_{k j}^{(i)}\left(\underline{\beta}_{j}^{(i)}\right)\right|_{\mathfrak{p}_{i j}}^{d\left(\mathfrak{p}_{i j}\right)}}{\left|\underline{\beta}_{j}^{(i)}\right|_{\mathfrak{p}_{i j}}^{d\left(\mathfrak{p}_{i j}\right)}}
$$


of all the linear forms is

$$
\ll H_{\mathbb{K}}(\underline{\beta})^{-3} c_{0}^{3\left(r_{n}+s_{n}\right)}\left(\prod_{\left|\beta_{j}\right|>1}\left|\beta_{j}\right|^{-\delta d_{j}(w-1) s_{n}} \cdot \prod_{j=1}^{l^{\prime}}|\beta|_{\mathfrak{p}_{j}}^{-(w-1) s_{n} d\left(\mathfrak{p}_{j}\right)}\right) .
$$

Since $w>1$, we infer from condition (ii) in the definition of a stammering sequence that there exists a positive real number $\eta$ such that

$$
2 \eta\left(r_{n}+s_{n}\right)<(w-1) s_{n}
$$

for every large positive integer $n$. Now, we choose $c_{0}$ close enough to 1 to ensure that $c_{1}:=H_{\mathbb{K}}(\beta) / c_{0}^{3 / \eta}$ satisfies $c_{1}>1$. Since $\delta>1$, we obtain

$$
\begin{aligned}
\Pi & \ll H_{\mathbb{K}}(\underline{\beta})^{-3} c_{0}^{3\left(r_{n}+s_{n}\right)}\left(\prod_{j=1}^{l}\left|\beta_{j}\right|^{d_{j}} \cdot \prod_{j=1}^{l^{\prime}}|\beta|_{\mathfrak{p}_{j}}^{d\left(\mathfrak{p}_{j}\right)}\right)^{-2 \eta\left(r_{n}+s_{n}\right)} \\
& \ll H_{\mathbb{K}}(\underline{\beta})^{-3}\left(c_{0}^{-3 / \eta} H_{\mathbb{K}}(\beta)\right)^{-\eta\left(r_{n}+s_{n}\right)}\left(\prod_{j=1}^{l}\left|\beta_{j}\right|^{d_{j}} \cdot \prod_{j=1}^{l^{\prime}}|\beta|_{\mathfrak{p}_{j}}^{d\left(\mathfrak{p}_{j}\right)}\right)^{-\eta\left(r_{n}+s_{n}\right)} \\
& \ll H_{\mathbb{K}}(\underline{\beta})^{-3}\left(c_{1} \cdot \prod_{j=1}^{l}\left|\beta_{j}\right|^{d_{j}} \cdot \prod_{j=1}^{l^{\prime}}|\beta|_{\mathfrak{p}_{j}}^{d\left(\mathfrak{p}_{j}\right)}\right)^{-\eta\left(r_{n}+s_{n}\right)} \cdot
\end{aligned}
$$

It thus follows from Lemma 2 that

$$
\Pi \ll H_{\mathbb{K}}(\underline{\beta})^{-3-\eta} .
$$

This shows that we have produced infinitely many algebraic solutions to inequality (4.1). Applying Theorem $\mathrm{S}$, we reach the conclusion that there exists a non-zero triple $\left(z_{1}, z_{2}, z_{3}\right)$ in $\mathbb{K}^{3}$ and an infinite set $\mathcal{N}$ of positive integers such that

$$
z_{1} \beta^{r_{n}+s_{n}}+z_{2} \beta^{r_{n}}+z_{3} P_{n}(\beta)=0
$$

for all $n \in \mathcal{N}$. Since $s_{n}$ tends to infinity with $n$, the number $z_{3} \in \mathbb{K}$ is non-zero. If $z_{1} \neq 0$, then dividing (5.9) by $\beta^{r_{n}+s_{n}}$ and letting $n$ tend to infinity along $\mathcal{N}$, we deduce that

$$
\lim _{n \rightarrow+\infty} \frac{P_{n}(\beta)}{\beta^{r_{n}+s_{n}}}=f_{\mathbf{a}}(\alpha)
$$

belongs to $\mathbb{K}$. If $z_{1}=0$, we divide (5.9) by $\beta^{r_{n}}$ and argue similarly to reach the same conclusion.

Given any integer $j$ with $2 \leq j \leq l$, we can replace $\alpha$ by $\alpha_{j}$ all along the above proof, and thus derive that $f_{\mathbf{a}, j}(\alpha)$ belongs to $\mathbb{L}\left(\alpha_{j}\right)$. The last statement about $f_{\mathbf{a}, j}(\alpha)=\sigma_{j}\left(f_{\mathbf{a}}(\alpha)\right)$ follows immediately by conjugating relation (5.9) by $\sigma_{j}$ for all $n \in \mathcal{N}$ and then arguing as above.

This concludes the proof of Theorem 1. 


\section{Proofs of Theorem 2 and Corollary 3}

Proof of Theorem 2. We may assume that $a_{0} \neq 0$, since otherwise we may eliminate $a_{0}$ and reindex the sequence (clearly, the new sequence obtained in this way is still stammering). Set

$$
\alpha=\sum_{k=0}^{+\infty} \frac{a_{k}}{b^{k}}
$$

Assume that $\alpha \in \overline{\mathbb{Q}}$. Since the complex conjugation is continuous, we get

$$
\bar{\alpha}=\sum_{k=0}^{+\infty} \frac{a_{k}}{\bar{b}^{k}} .
$$

Hence, $\bar{\alpha} \in \overline{\mathbb{Q}}$. By Theorem 1 , we find that $\alpha \in \mathbb{Q}[b]$. In fact, Theorem 1 shows that more is true, namely (we keep the notation used in the proof of this theorem) that there exist a non-zero triple $\left(z_{1}, z_{2}, z_{3}\right)$ in $\mathbb{K}^{3}=\mathbb{Q}[b]^{3}$ and an infinite set of positive integers $\mathcal{N}_{1}$ such that

$$
z_{1} b^{r_{n}+s_{n}}-z_{2} b^{r_{n}}-z_{3} P_{n}(b)=0
$$

for every $n \in \mathcal{N}_{1}$. Here,

$$
P_{n}(X)=\sum_{k=0}^{r_{n}-1} a_{k} X^{r_{n}-k}\left(X^{s_{n}}-1\right)+\sum_{k=0}^{s_{n}-1} a_{r_{n}+k} X^{s_{n}-k} \in \mathbb{Z}[X],
$$

as in Lemma 1. Dividing both sides of (6.1) by $b^{r_{n}+s_{n}}$ and letting $n$ tend to infinity along $\mathcal{N}_{1}$, we get $z_{1}=z_{3} \alpha$. Hence, if $z_{3}=0$, then $z_{1}=0$, and now (6.1) shows that $z_{2}=0$ as well, which is impossible.

Thus, $z_{3} \neq 0$ and by multiplying both sides of (6.1) by $z_{3}^{-1}$, it follows that we may assume that $z_{3}=1$. Since $z_{1}=z_{3} \alpha$, we get $z_{1}=\alpha$. Inserting this into (6.1) and dividing both sides of the resulting relation by $b^{r_{n}+s_{n}}$ we get

$$
\alpha-\frac{z_{2}}{b^{s_{n}}}=\frac{P_{n}(b)}{b^{r_{n}+s_{n}}}=\sum_{k=0}^{s_{n}+r_{n}-1} \frac{a_{k}}{b^{k}}-\sum_{k=0}^{r_{n}-1} \frac{a_{k}}{b^{s_{n}+k}} .
$$

Substituting the formula for $\alpha$, performing the obvious cancellations and multiplying both sides of the resulting formula by $b^{s_{n}}$, we get

$$
z_{2}=\sum_{k=0}^{r_{n}-1} \frac{a_{k}}{b^{k}}+\sum_{k \geq r_{n}} \frac{a_{s_{n}+k}}{b^{k}} .
$$

Since the above relation is true for every $n$ in $\mathcal{N}_{1}$, it follows easily that $\alpha=z_{2}$ because the right hand side of the above relation tends to $\alpha$ when $n$ tends to infinity along $\mathcal{N}_{1}$. Replacing $z_{1}=z_{2}=\alpha$ in (6.1), we get

$$
\alpha b^{r_{n}}\left(b^{s_{n}}-1\right)=P_{n}(b)
$$

for every $n \in \mathcal{N}_{1}$. 
Assume first that $\alpha=0$. We then get $P_{n}(b)=0$. Note that

$$
P_{n}(X)=\sum_{k=0}^{r_{n}+s_{n}-1} c_{k} X^{r_{n}+s_{n}-k},
$$

where $c_{k}=a_{k}$ if $k=0, \ldots, s_{n}-1$, and $c_{k}=a_{k}-a_{k-s_{n}}$ if $k=s_{n}, \ldots, r_{n}+$ $s_{n}-1$. Since $a_{0} \neq 0$, it follows that $P_{n}(X)$ is not the zero polynomial. Note that each of its coefficients is either of the form $a_{i}$, or of the form $a_{j}-a_{h}$ for some appropriate indices $i, j$ and $h$. Dividing both sides of equation (6.2) by an appropriate power of $b$ to emphasize a constant term (i.e., a term which is not a multiple of $b$ ), we get a relation of the form

$$
c_{0, n} b^{d_{n}}+c_{1, n} b^{d_{n}-1}+\cdots+c_{d_{n}, n}=0,
$$

where $d_{n}>0$, the coefficients $c_{j, n}$ are integers, $c_{0, n}=a_{0} \neq 0, c_{d_{n}, n} \neq 0$, and the absolute values of all coefficients $c_{i, n}$ do not exceed $|b|^{2}-1$. However, the above relation implies that the polynomial

$$
Q_{n}(X)=c_{0, n} X^{d_{n}}+c_{1, n} X^{d_{n}-1}+\cdots+c_{d_{n}, n} \in \mathbb{Z}[X]
$$

is a multiple of the minimal polynomial of $b$ over $\mathbb{Z}$. Since the constant term of the minimal polynomial over $\mathbb{Z}$ of $b$ is $|b|^{2}=\bar{b} \cdot b$, and this number must divide the non-zero integer $c_{d_{n}, n}$ of absolute value $\leq|b|^{2}-1$, we get a contradiction.

From now on, we assume that $\alpha \neq 0$.

Let $n_{1}<n_{2}<\cdots$ be an infinite sequence of positive integers in $\mathcal{N}_{1}$. Thus, relation (6.2) holds for $n=n_{i}$ with some $i \geq 1$. The sequence $\left(r_{n}\right)_{n \geq 1}$ might be bounded or not. If it is unbounded, we may assume, up to discarding some values for the $n_{i}$ 's, that $r_{n_{i+1}}>s_{n_{i}}+r_{n_{i}}$ for all $i \geq 1$, while if it is bounded, we may assume that $r_{n_{i}}$ is a constant for all $i \geq 1$. We also assume that $s_{n_{i+1}}>r_{n_{i}}+s_{n_{i}}$ for all $i \geq 1$. This is possible because the sequence $\left(s_{n}\right)_{n \geq 1}$ is increasing. From now on, we write $m=n_{j}$ and $n=n_{i}$ for some $j>i \geq 1$. Dividing the two relations (6.2) obtained for $m$ and $n$, we get

$$
\frac{b^{r_{m}}\left(b^{s_{m}}-1\right)}{b^{r_{n}}\left(b^{s_{n}}-1\right)}=\frac{P_{m}(b)}{P_{n}(b)}
$$

which gives

$$
b^{r_{m}-r_{n}}\left(b^{s_{m}}-1\right) P_{n}(b)-\left(b^{s_{n}}-1\right) P_{m}(b)=0 .
$$

The expression appearing on the left hand side of the last equation is a polynomial in $b$ (note that $r_{m} \geq r_{n}$ ). Put $D=\left(r_{m}-r_{n}\right)+s_{m}+r_{n}+s_{n}=$ $r_{m}+s_{m}+s_{n}$. Note that since $a_{0} \neq 0$, it follows that $D$ is the common degree of the polynomials $X^{r_{m}-r_{n}}\left(X^{s_{m}}-1\right) P_{n}(X)$ and $\left(X^{s_{n}}-1\right) P_{m}(X)$. We show that if $n$ is fixed and $m$ is very large, then one of the polynomials

$$
Q_{m, n}(X)=X^{r_{m}-r_{n}}\left(X^{s_{m}}-1\right) P_{n}(X)-\left(X^{s_{n}}-1\right) P_{m}(X) \in \mathbb{Z}[X]
$$


is not the zero polynomial, and that $Q_{m, n}(X)$ is of the form $X^{\lambda} R_{m, n}(X)$, where $R_{m, n}(0)$ is non-zero and of the form $\pm a_{i}$ or $a_{j}-a_{h}$ for some appropriate indices $i, j$ and $h$, which leads to the same final contradiction as in the case when $\alpha=0$.

To see that $Q_{m, n}(X)$ is non-zero, note that the coefficient of $X^{D-r_{n}-s_{n}-t}$, where $t=0,1, \ldots, s_{m}-s_{n}-r_{n}$, is simply $a_{s_{n}+t}-a_{t}$. If this coefficient is zero for all $t$ in the above range and for arbitrarily large values of $m$, we then infer that the sequence $\left(a_{k}\right)_{k \geq 0}$ is periodic with period $s_{n}$, which is a contradiction.

We now look at the last non-zero coefficient of $Q_{m, n}(X)$. Note that if $r_{m}-r_{n}>s_{n}$, then that coefficient is $a_{r_{m}+s_{m}-1}-a_{r_{m}-1}$, and it is the coefficient of $X$. If $a_{r_{m}+s_{m}-1}-a_{r_{m}-1}$ is non-zero, we have already obtained the desired contradiction. If not, we divide both sides of (6.3) by $X$ and work with the polynomial $Q_{m, n}(X) / X$. Continuing in this way $r_{m}-r_{n}$ times, we may assume that $a_{s_{m}+r_{m}-t}=a_{r_{m}-t}$ for all $t=1, \ldots, r_{m}-r_{n}$. At this point, $Q_{m, n}(X)$ is replaced by the polynomial

$$
Q_{m, n}(X) / X^{r_{m}-r_{n}}=\left(X^{s_{m}}-1\right) P_{n}(X)-\left(X^{s_{n}}-1\right) P_{m}(X) / X^{r_{m}-r_{n}} .
$$

Note that if $r_{m}=r_{n}$, then the above discussion is unnecessary and the polynomial (6.3) is just $Q_{m, n}(X)$. In what follows, we study the above polynomial. Note that

$$
\begin{aligned}
S_{m, n}(X)= & \left(X^{s_{m}}-1\right) P_{n}(X) \\
= & a_{0} X^{s_{m}+r_{n}+s_{n}}+a_{1} X^{s_{m}+r_{n}+s_{n}-1}+\cdots \\
& +a_{s_{n}-1} X^{s_{m}+r_{n}+1}+\left(a_{s_{n}}-a_{0}\right) X^{s_{m}+r_{n}}+\cdots \\
& +\left(a_{s_{n}+r_{n}-1}-a_{r_{n}-1}\right) X^{s_{m}+1}-a_{0} X^{r_{n}+s_{n}}+\cdots \\
& -a_{s_{n}-1} X^{r_{n}+1}-\left(a_{s_{n}}-a_{0}\right) X^{r_{n}}-\cdots-\left(a_{s_{n}+r_{n}-1}-a_{r_{n}-1}\right) X .
\end{aligned}
$$

Thus, with $D_{1}=s_{m}+s_{n}+r_{n}$, it follows that

$$
S_{m, n}(X)=\sum_{k=0}^{D_{1}-1} c_{k} X^{D_{1}-k},
$$

where $c_{k}$ is given by:

(i) $a_{k}$ if $k=0, \ldots, s_{n}-1$;

(ii) $a_{k}-a_{k-s_{n}}$ if $k=s_{n}, \ldots, s_{n}+r_{n}-1$;

(iii) 0 if $k=s_{n}+r_{n}, \ldots, s_{m}-1$;

(iv) $-a_{k-s_{m}}$ if $k=s_{m}, \ldots, s_{m}+s_{n}-1$;

(v) $-\left(a_{k-s_{m}}-a_{k-s_{m}-s_{n}}\right)$ if $k=s_{m}+s_{n}, \ldots, s_{m}+s_{n}+r_{n}-1$.

For the second polynomial appearing in (6.3),

$$
T_{m, n}(X)=\left(X^{s_{n}}-1\right) P_{m}(X) / X^{r_{m}-r_{n}},
$$


a similar calculation gives

$$
T_{m, n}(X)=\sum_{k=0}^{D_{1}-1} d_{k} X^{D_{1}-k},
$$

where the coefficients $d_{k}$ are given by:

(i) $a_{k}$ if $k=0, \ldots, s_{n}-1$;

(ii) $a_{k}-a_{k-s_{n}}$ if $k=s_{n}, \ldots, s_{m}-1$;

(iii)' $a_{k}-a_{k-s_{m}}-a_{k-s_{n}}$ if $k=s_{m}, \ldots, s_{m}+r_{n}-1$;

$(\text { iv })^{\prime}-a_{k-s_{n}}$ if $k=s_{m}+r_{n}, \ldots, s_{m}+s_{n}-1$;

$(\mathrm{v})^{\prime}-\left(a_{k-s_{n}}-a_{k-s_{n}-s_{m}}\right)$ if $k=s_{m}+s_{n}, \ldots, s_{m}+s_{n}+r_{n}-1$,

provided that $s_{n}>r_{n}$. When $s_{n}=r_{n}$, the same holds except that (iv)' is vacuous, while if $s_{n}<r_{n}$, then the group (iii) $)^{\prime}-(\mathrm{v})^{\prime}$ above should be replaced by

(iii)" $a_{k}-a_{k-s_{m}}-a_{k-s_{n}}$ if $k=s_{m}, \ldots, s_{m}+s_{n}-1$;

(iv) ${ }^{\prime \prime} a_{k}-a_{k-s_{m}}-\left(a_{k-s_{n}}-a_{k-s_{n}-s_{m}}\right)$ if $k=s_{m}+s_{n}, \ldots, s_{m}+r_{n}-1$;

$(\mathrm{v})^{\prime \prime}-\left(a_{k-s_{n}}-a_{k-s_{n}-s_{m}}\right)$ if $k=s_{m}+r_{n}, \ldots, s_{m}+r_{n}+s_{n}-1$.

It is now easy to obtain the list of coefficients of $Q_{m, n}(X)$. Namely, $c_{k}-d_{k}$ equals (independently of whether $s_{n}>r_{n}, s_{n}=r_{n}$ or $s_{n}<r_{n}$ ):

(i) 0 if $k=0,1, \ldots, s_{n}+r_{n}-1$;

(ii) $-\left(a_{k}-a_{k-s_{n}}\right)$ if $k=s_{n}+r_{n}, \ldots, s_{m}+r_{n}-1$;

(iii) $-\left(a_{k-s_{m}}-a_{k-s_{n}}\right)$ if $k=s_{m}+r_{n}, \ldots, s_{m}+s_{n}+r_{n}-1$,

which confirms the claim about the coefficients of $Q_{m, n}(X)$. Thus, Theorem 2 is proved.

Proof of Corollary 3. Let $\mathbf{a}^{\prime}$ be the sequence $a_{0}, 0, a_{1}, 0, a_{2}, 0, \ldots$ It is clear that $\mathbf{a}^{\prime}$ is also stammering. Now the result follows by applying Theorem 2 to the pair $\left(\mathbf{a}^{\prime}, b^{1 / 2} i\right)$.

Acknowledgments. We are very grateful to the referee for having pointed to us one important mistake and for many valuable suggestions. F. L. thanks Professor Hans Peter Schlickewei for useful advice. This paper was started during a visit of F. L. at the University Strasbourg 1 in July 2005. He warmly thanks the mathematical department for their hospitality. Y. B. and F. L. were supported in part by the joint Project France-Mexico ANUIES-ECOS M01-M02.

\section{References}

[1] B. Adamczewski and Y. Bugeaud, On the complexity of algebraic numbers I. Expansions in integer bases, Ann. of Math. 165 (2007), 547-565. 
[2] B. Adamczewski and Y. Bugeaud, Dynamics for $\beta$-shifts and Diophantine approximation, Ergodic Theory Dynam. Systems 27 (2007), 1695-1711.

[3] B. Adamczewski, Y. Bugeaud et F. Luca, Sur la complexité des nombres algébriques, C. R. Math. Acad. Sci. Paris 339 (2004), 11-14.

[4] J.-P. Allouche and J. O. Shallit, Automatic Sequences: Theory, Applications, Generalizations, Cambridge Univ. Press, Cambridge, 2003.

[5] P. Corvaja and U. Zannier, Some new applications of the subspace theorem, Compos. Math. 131 (2002), 319-340.

[6] K. Mahler, Arithmetische Eigenschaften der Lösungen einer Klasse von Funktionalgleichungen, Math. Ann. 101 (1929), 342-366.

[7] —, Lectures on Diophantine Approximation, Part 1: g-adic Numbers and Roth's Theorem, Univ. of Notre Dame, Ann Arbor, 1961.

[8] K. Nishioka, Mahler Functions and Transcendence, Lecture Notes in Math. 1631, Springer, Berlin, 1996.

[9] H. P. Schlickewei, The p-adic Thue-Siegel-Roth-Schmidt theorem, Arch. Math. (Basel) 29 (1977), 267-270.

[10] W. M. Schmidt, Diophantine Approximations and Diophantine Equations, Lecture Notes in Math. 1467, Springer, Berlin, 1991.

[11] M. Waldschmidt, Diophantine Approximation on Linear Algebraic Groups, Grundlehren Math. Wiss. 326, Springer, Berlin, 2000.

CNRS, Université de Lyon, Université Lyon 1

Institut Camille Jordan

43 boulevard du 11 novembre 1918

69622 Villeurbanne Cedex, France

E-mail: Boris.Adamczewski@math.univ-lyon1.fr

Mathematical Institute

UNAM

Ap. Postal 61-3 (Xangari), CP 58089

Morelia, Michoacán, Mexico

E-mail: fluca@matmor.unam.mx
U.F.R. de mathématiques Université Louis Pasteur 7 , rue René Descartes 67084 Strasbourg Cedex, France E-mail: bugeaud@math.u-strasbg.fr

Received on 12.4.2006

and in revised form on 30.4.2008 